\title{
UNSTEADY RESPONSE OF FLOW SYSTEM AROUND BALANCE PISTON IN A ROCKET PUMP
}

\author{
S. Kawasaki ${ }^{1}$, T. Shimura ${ }^{1}$, M. Uchiumi ${ }^{1}$, M. Hayashi ${ }^{2}$, \\ and J. Matsui ${ }^{3}$ \\ 1 Japan Aerospace Exploration Agency \\ 1, Koganezawa, Kimigaya, Kakuda, Miyagi, Japan \\ ${ }^{2}$ IHI Corporation \\ 1, Shin-Nakahara-Cho, Isogo-ku, Yokohama, Japan \\ ${ }^{3}$ Yokohama National University \\ 79-5, Tokiwadai, Hodogaya-ku, Yokohama, Japan
}

In the rocket engine turbopump, a self-balancing type of axial thrust balancing system using a balance piston is often applied. In this study, the balancing system in liquid-hydrogen $\left(\mathrm{LH}_{2}\right)$ rocket pump was modeled combining the mechanical structure and the flow system, and the unsteady response of the balance piston was investigated. The axial vibration characteristics of the balance piston with a large amplitude were determined, sweeping the frequency of the pressure fluctuation on the inlet of the balance piston. This vibration was significantly affected by the compressibility of $\mathrm{LH}_{2}$.

\section{NOMENCLATURE}

$\begin{array}{ll}f & \text { frequency of axial vibration, } \mathrm{Hz} \\ f_{n} & \text { natural frequency, } \mathrm{Hz} \\ F & \text { axial thrust, } \mathrm{N} \\ F^{*}=F /\left(\pi R_{\mathrm{im}}^{2} p_{\text {in }}\right) & \text { normalized axial thrust } \\ K & \text { stiffness of balance piston, } \mathrm{N} / \mathrm{m} \\ M & \text { mass of rotor assembly, } \mathrm{kg} \\ p & \text { pressure, } \mathrm{Pa} \\ p^{*}=p / p_{\mathrm{in}} & \text { normalized pressure } \\ R_{\mathrm{im}} & \text { radius of impeller, } \mathrm{m} \\ S & \text { clearance of orifice, } \mathrm{m} \\ S^{*}=S /\left(S_{1}+S_{2}\right) & \text { normalized clearance of orifice } \\ T & \text { temperature of } \mathrm{LH}_{2}, \mathrm{~K}\end{array}$




\section{Subscripts}

bp balance piston or balance piston chamber

in inlet of balance piston (discharge of impeller)

oth other than balance piston

1, 2 inlet orifice (\#1) and outlet orifice (\#2) of balance piston, respectively

\section{INTRODUCTION}

Usually, a rocket engine turbopump must be operated at a very high rotating speed to deliver the high-pressure liquid propellant to the combustor. Consequently, large axial thrust is generated on the rotor assembly of the turbopump. Therefore, it is essential to reduce or balance the axial thrust for the reliability of bearings supporting the rotor assembly. The self-balancing type axial thrust balancing system using balance piston is often applied in the rocket engine turbopump. In designing this balancing system, accurate prediction of the axial thrust characteristics is very important.

Figure 1 shows a typical rocket engine turbopump configuration and its internal flow paths. The rotor assembly is allowed to move in the axial direction and shift to the balancing point automatically. However, too high axial vibration not only destroys bearings but also causes metal to metal rubbing which can cause an explosion. To avoid these problems, it would be helpful for the design of the rocket engine turbopump to simulate the unsteady response of the balancing system and clarify the vibration mechanism.

Kurokawa et al. [1] examined the axial thrust behavior in a rocket pump calculated by steady theoretical analysis of the internal flow. The analysis code developed with this theoretical analysis was applied to investigate the axial thrust characteristics [2, 3]. Della Gatta et al. [4] investigated the axial thrust balance by steady computational fluid dynamics (CFD) analysis. The obtained results suggest that the methods to predict steady axial thrust can be used in turbopump designing. Furthermore, transient axial thrust was calculated during start transient of a rocket turbopump by Hooser et al. [5]. This code was extended to

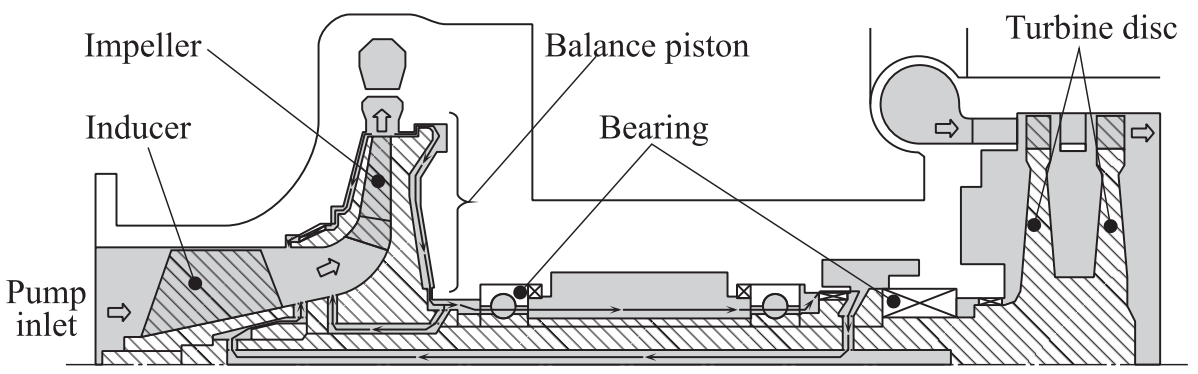

Figure 1 An example of the rocket $\mathrm{LH}_{2}$ pump and internal flow paths 
the model of transient flow to predict time-dependent flow characteristics and a good agreement was observed between measurements and predictions.

Dynamic characteristics of the clearance flow have also been investigated. Childs [6] and Hsu and Brennen [7] have examined the dynamic characteristics of flows in the clearance between the casing and front shroud of impellers by the bulk flow model. By experiments and computations, the dynamic characteristics of the flow between stationary and rotating disks under axial oscillation have been examined by Horiguchi et al. [8]. However, the unsteady response of the balance piston, which is an important device for the axial thrust balance, has not been clarified.

In the present study, the balancing system around the balance piston was modeled combining the mechanical structure and the flow system, and the unsteady response of the balance piston was investigated.

\section{BALANCE PISTON}

Figure 2 shows an axial thrust self-balancing system using a balance piston. The balance piston consists of two orifices and a chamber surrounded by these two orifices. The inlet orifice $(\# 1)$ is located at the impeller outlet and the outlet orifice (\#2) is located at the small-radius position of the back shroud. The rotor assembly is allowed to move in the axial direction to control the clearances of the orifices. If unbalance axial thrust is imposed on the rotor assembly in the direction from the pump inlet side toward the turbine part, the rotor assembly moves toward the turbine side. As a result, the clearance of orifice \#1 increases and that of orifice \#2 decreases. This causes the pressure in the balance piston chamber to increase, hence the axial thrust in the direction from the turbine part toward the pump inlet side increases. In this way, unbalance axial thrust imposed on the rotor assembly can be compensated automatically.

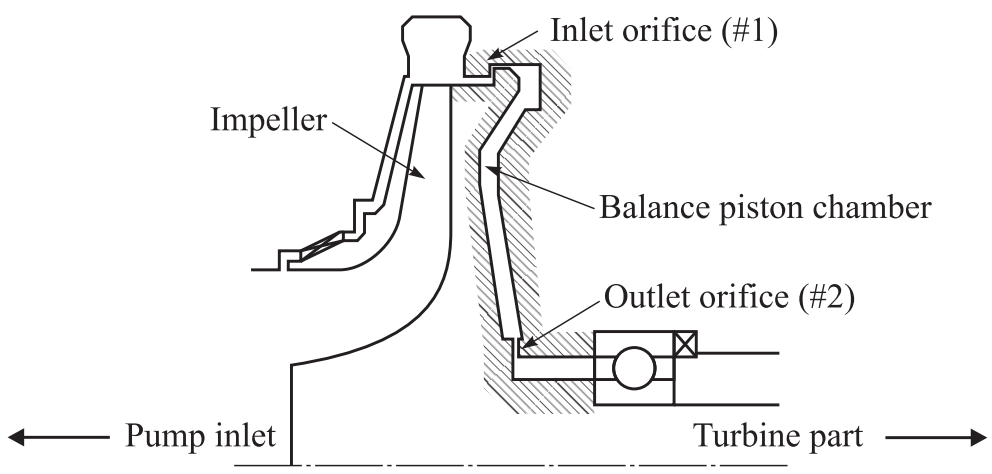

Figure 2 Configuration of balance piston 
In the balance piston chamber, the radial pressure distribution is mainly determined by the centrifugal force effect caused by the swirl in the chamber. The axial thrust generated by the balance piston can be calculated by integration of that radial pressure distribution.

\section{CALCULATION METHOD}

In the previous studies $[2,3]$, the axial thrust characteristics were calculated by in-house one-dimensional (1D) flow analysis code, which takes account of momentum transported into the chambers and the boundary layer. In this code, it is assumed that the flow is steady and incompressible. In the present study, the axial balancing system was modeled and calculated using a $1 \mathrm{D}$ multidomain system simulation tool, AMESim (Rev. 10) [9], which can simulate both incompressible and compressible flows in unsteady state. This tool had been previously used for transient modeling and the simulation of cryogenic rocket engine by Rhote-Vaney et al. [10]. In the study, the engine system had been presented by connecting submodels, and the dynamics of the whole engine system had been simulated. However, the dynamics of the turbopump itself had not yet been clarified.

Figure 3 shows the model in which the balancing system of a typical $\mathrm{LH}_{2}$ pump (see Fig. 1) is expressed. The model consists of the mechanical structure of the balance piston and the flow path along the back side of the impeller. The balance piston is pushed by the axial thrust generated by the pressure in the balance piston and pulled by that generated in locations other than the balance piston. The orifice clearances are connected with the displacement of balance piston by the function component (1). The volume of the balance piston chamber is varied with the balance piston displacement. This chamber model is not able to treat the pressure distribution inside the chamber, although the balance piston chamber has the radial pressure distribution. In this model, the equivalent radial pressure drop estimated by the integration of the pressure distribution, which is calculated by steady analysis of the internal flow [3], is given to this chamber model by the function component (2). The downstream path of orifice \#2 is expressed by a simple model (chamber, restriction and tank component). In order to investigate the unsteady response of the balancing system, the pressure fluctuation on the inlet of the balance piston was input. The fluctuation frequency was swept from $0 \mathrm{~Hz}$ to $2000 \mathrm{~Hz}$ and the fluctuation amplitude was $10 \%$ of the inlet pressure of the balance piston. Other calculating conditions are described as follows:

- thermal fluid properties of $\mathrm{LH}_{2}$ were given by polynomials approximation;

- total clearance of orifices \#1 and \#2 was axially about $0.3 \mathrm{~mm}$; 


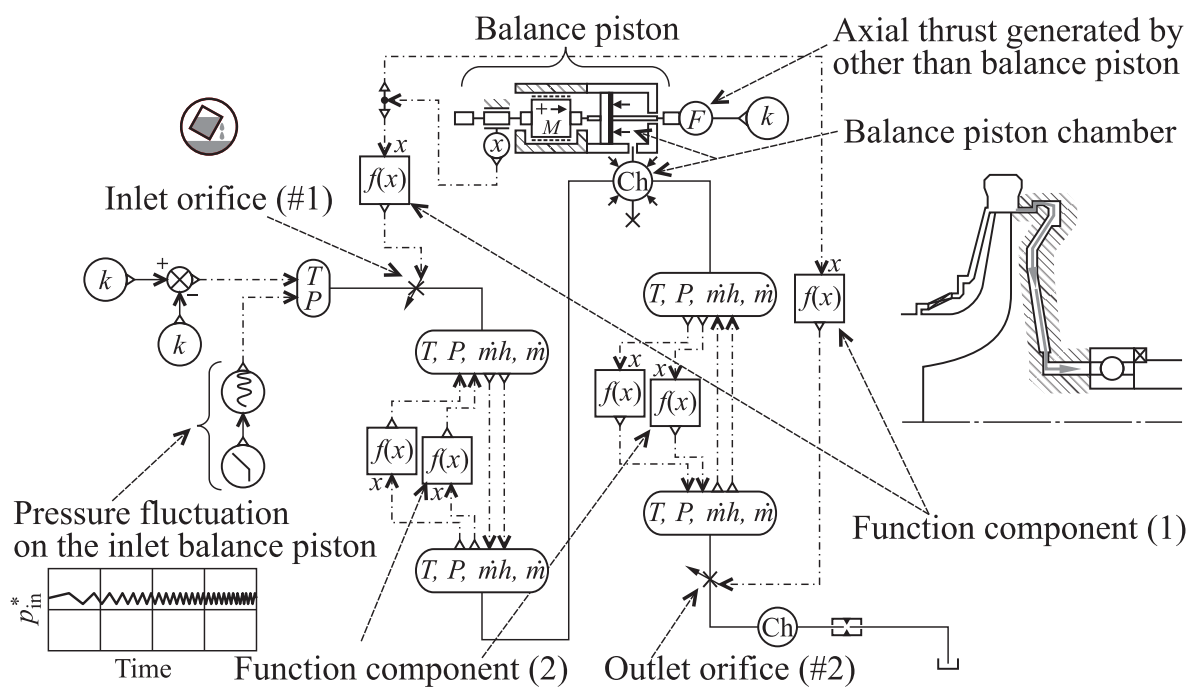

Figure 3 Model structure of internal flow system around balance piston

- rotating speed was 50,000 rpm (rotating speed was not used in this-code directly, but was used in the steady analysis code);

- temperature in the inlet of the balance piston was $28 \mathrm{~K}$ unless otherwise indicated;

- heat caused by the disk friction of the impeller was not considered;

- heat input from outside of turbopump was not considered;

- cavitation was not considered; and

- damping caused by the balancing system was not considered.

\section{RESULTS}

First of all, to verify that the present model could achieve balance at proper positions, the steady-state calculation by this model was carried out and compared with the results of the steady analysis of the internal flow [3]. Figure 4 shows static axial thrust characteristics of balance piston. The horizontal axis shows the normalized clearance of orifice \#1 and the vertical axis shows the axial thrust generated by the balance piston. The positive direction of the thrust was from the turbine part to the pump inlet side. The result of the present code showed good agreement with that of the steady-analysis code. The equivalent radial pressure drop in the balance piston chamber was given by the result of the steady analysis 


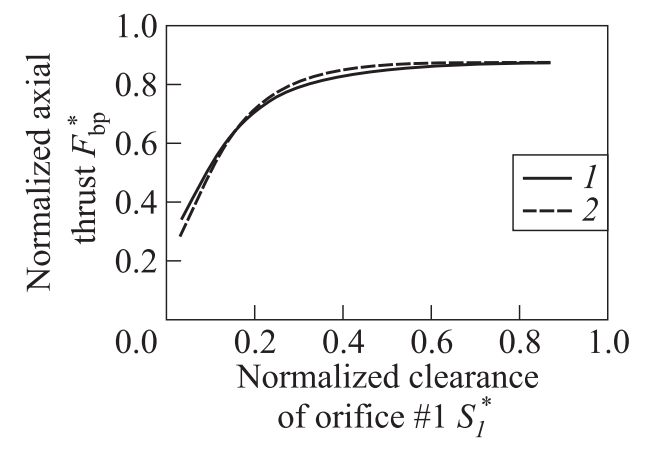

Figure 4 Static axial thrust curves of balance piston: 1 - present code; and 2 steady-analysis code pressure drop of orifice \#1 and orifice \#2. Without damping, the natural frequency of the balance piston is expressed as follows:

$$
f_{n}=\frac{1}{2 \pi} \sqrt{\frac{K}{M}} .
$$

By inputting the pressure fluctuation on the inlet of the sweeping frequency of the balance piston, the unsteady response of the balance piston was calculated by the present model. Figure $5 a$ shows the clearance of orifice \#1 and the pressure of the balance piston chamber to show the behavior of the balance piston. Figure $5 b$ shows the fast Fourier transform (FFT) results of these data. The large amplitude vibration of the balance piston and the pressure fluctuation in the chamber were determined at about $1200 \mathrm{~Hz}$. The mean normalized

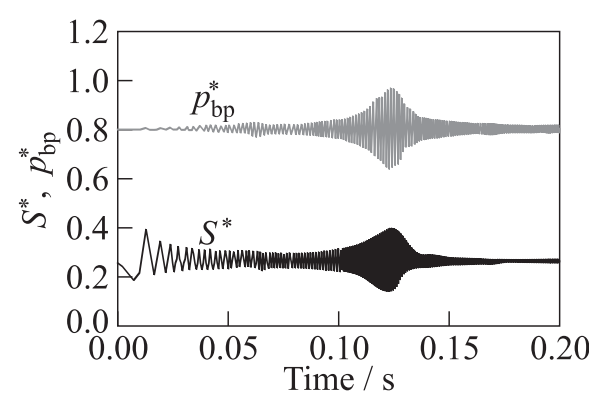

(a)

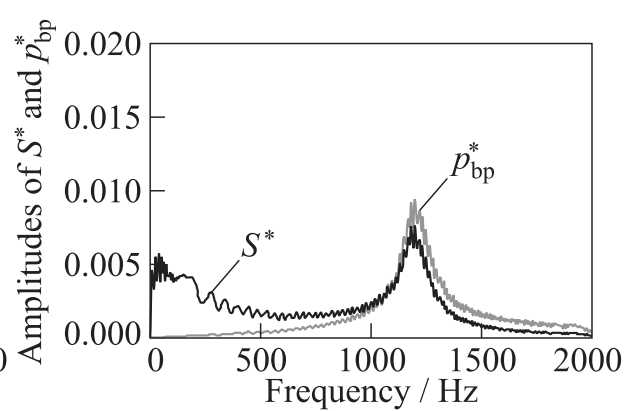

(b)

Figure 5 Calculation results with base condition $\left(p_{\mathrm{bp}}^{*}-\right.$ normalized pressure in balance piston chamber; and $S^{*}$ - normalized clearance of orifice \#1): (a) time series and (b) FFT results 


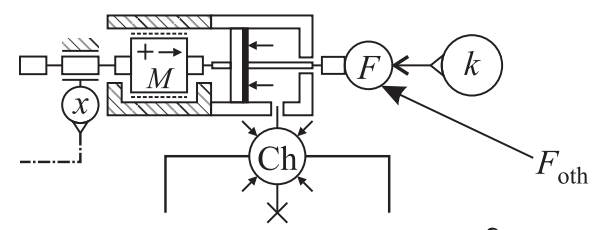

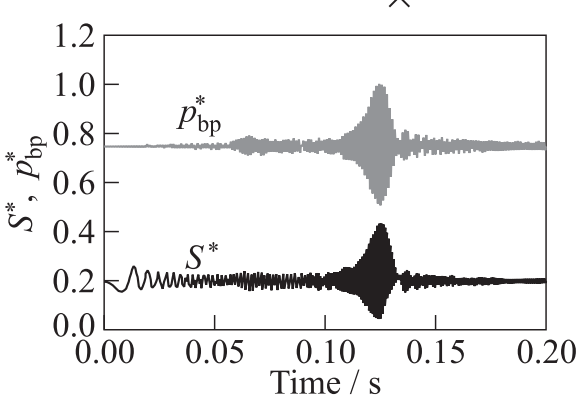

(a)

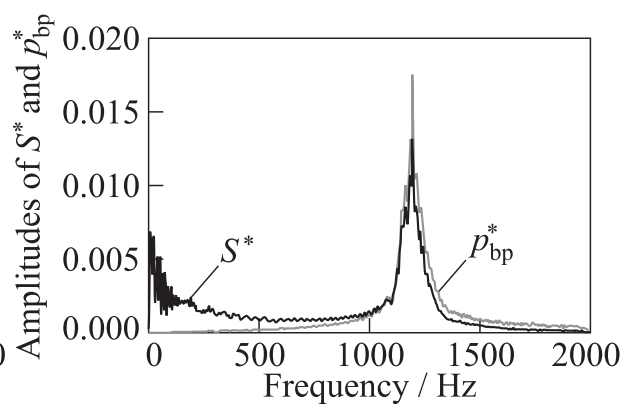

(b)

Figure 6 Calculation results with $7 \% F_{\text {oth }}$ (axial thrust generated by other than the balance piston) less than that of the base condition: $(a)$ time series and (b) FFT results

clearance of orifice \#1 was about 0.27 and the natural frequency of the balance piston was $900 \mathrm{~Hz}$ which was calculated by the static axial thrust characteristics of the balance piston and Eq. (1). There is a slight difference of the vibration frequency with large amplitude.

In order to investigate the effects of the static stiffness of the balance piston, the axial thrust generated in the locations other than the balance piston, $F_{\text {oth }}$, was decreased. With the reduction of $F_{\text {oth }}$, the static balancing point was moved toward the pump inlet side. The calculation result is shown in Fig. 6. According to the static axial thrust characteristics of the balance piston (see Fig. 4), the stiffness of the balance piston is expected to be larger and the natural frequency is expected to be higher when the orifice \#1 clearance is decreased. In the results shown in Figs. 5 and 6 , the mean normalized orifice \#1 clearance became 0.26 and 0.2 and the natural frequency calculated by the characteristic curve of the static axial thrust became 900 and $1200 \mathrm{~Hz}$, respectively. However, the peak vibration frequencies of the two results shown in Figs. 5 and 6 were not so different (about $1200 \mathrm{~Hz}$ ). This means that these vibrations were not only determined by the static stiffness slope of the curve, but were affected by other factors.

As another factor, the compressibility of $\mathrm{LH}_{2}$ is conceivable, because the bulk modulus of $\mathrm{LH}_{2}$ is considerably smaller than that of a typical liquid. As the bulk modulus of $\mathrm{LH}_{2}$ is quite variable depending on the temperature, the calculation was carried out by the condition at a temperature different from that of the 


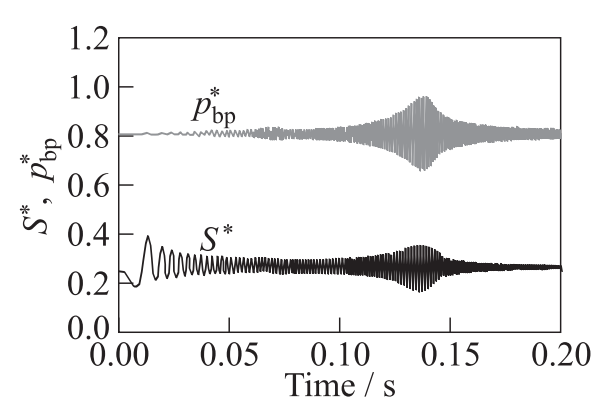

(a)

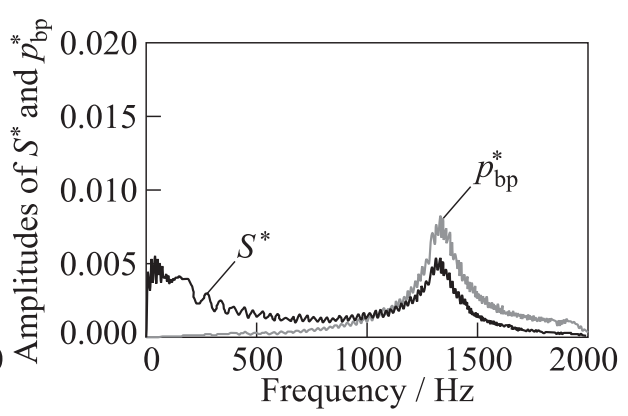

(b)

Figure 7 Calculation results with $4 \mathrm{~K} T_{\text {in }}$ lower than that of the base condition: (a) time series and (b) FFT results

base condition. The calculation result is shown in Fig. 7, when the temperature on the inlet of the balance piston, $T_{\mathrm{in}}$, decreased to $24 \mathrm{~K}$. The axial vibration with large amplitude was determined at about $1400 \mathrm{~Hz}$ which was $200 \mathrm{~Hz}$ higher than that of the base condition. The bulk modulus of $\mathrm{LH}_{2}$ at the temperature of $24 \mathrm{~K}$ is 1.38 times larger than that at the temperature of $28 \mathrm{~K}$. The natural frequency of the liquid is proportional to the square root of the bulk modulus, so that the natural frequency of $\mathrm{LH}_{2}$ at the temperature of $24 \mathrm{~K}$ is 1.2 times higher than that at the temperature of $28 \mathrm{~K}$. The peak vibration frequency of the case at $T_{\text {in }}=24 \mathrm{~K}$ is 1.2 times higher than that at $T_{\mathrm{in}}=28 \mathrm{~K}$. This means that the large vibration of the balance piston was affected significantly by the compressibility of $\mathrm{LH}_{2}$.

If the balance piston chamber volume increases, the stiffness of the liquid in the balance piston decreases. Figure 8 shows the calculation results when the volume of the balance piston chamber, $V_{\mathrm{bp}}$, was $50 \%$ larger than that of the base condition by extending the chamber clearance. The axial vibration with a large amplitude was determined at about $1000 \mathrm{~Hz}$, which was $200 \mathrm{~Hz}$ lower than that of the base condition, and the peak amplitude of the vibration was about twice that of the base condition. The characteristic curve of the static axial thrust of this case was the same as that of the base condition as shown in Figs. 5 and 9. This means that the difference between these large vibrations was not due to the static axial thrust characteristics but rather due to the compressibility of $\mathrm{LH}_{2}$.

When the volume of the balance piston chamber is larger, the amplitude of the axial vibration due to the liquid compressibility is larger. To restrain the axial vibration, the balance piston chamber volume should be as low as possible for the high compressible liquid in designing the balance piston. Developing that idea, there are some possibilities to control the axial vibration using the liquid compressibility like an accumulator. For that purpose, it is necessary to develop a more accurate model. 


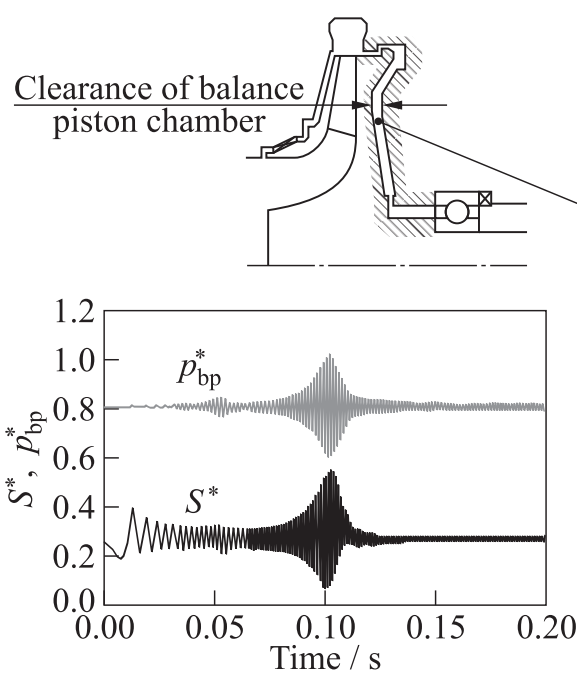

(a)
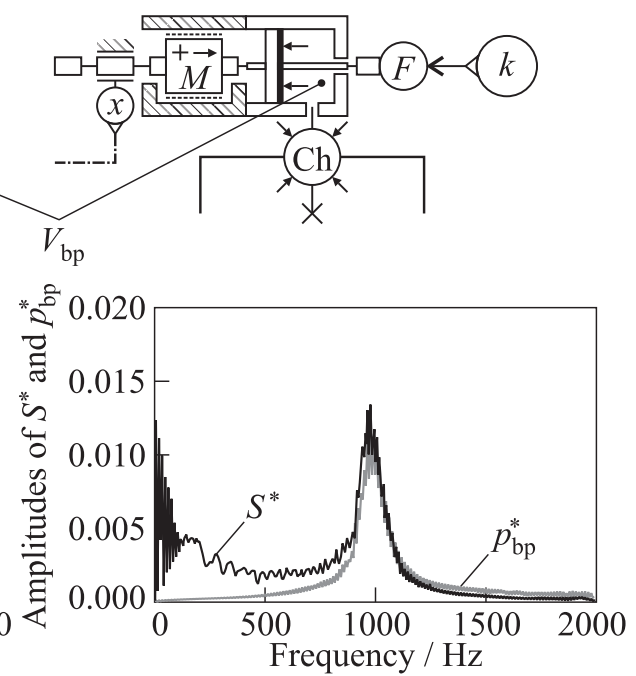

(b)

Figure 8 Calculation results with $50 \% V_{\mathrm{bp}}$ (volume of the balance piston chamber) larger than that of the base condition: $(a)$ time series and (b) FFT results

Furthermore, it is necessary for an accurate simulation to take account of the influence of a temperature change due to the heat caused by the disk friction and so on. If the local temperature and pressure reach saturating conditions, the internal flow will be transformed into two-phase cavitating flow. In such conditions, it must be noticed that the unsteady response of the balance piston could be different from that in the one-phase liquid flow.

Finally, the calculation time of each case is about 10 seconds by a typical personal computer.

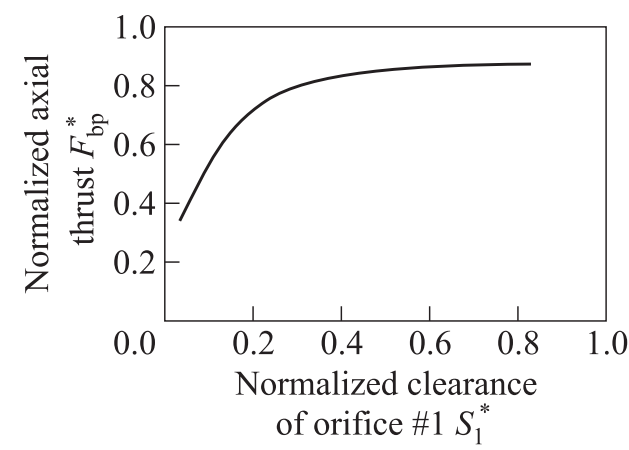

Figure 9 Static axial thrust curve of balance piston with $50 \% V_{\mathrm{bp}}$ larger than that of the base condition

\section{CONCLUDING REMARKS}

The internal flow system around the balance piston in an $\mathrm{LH}_{2}$ rocket pump was modeled and the unsteady response of balance piston was investigated, inputting 
the pressure fluctuation on the inlet of the balance piston. The main conclusions are summarized as follows:

(1) the balancing system could be modeled combining the mechanical structure and the flow system;

(2) sweeping the frequency of the pressure fluctuation on the inlet of the balance piston, the axial vibration of the balance piston with large amplitude was determined;

(3) this large vibration was affected significantly by the compressibility of $\mathrm{LH}_{2}$. Therefore, the vibration is influenced by the bulk modulus of $\mathrm{LH}_{2}$ and the volume of the balance piston chamber; and

(4) this unsteady code is able to estimate the axial vibration frequency of the balance piston taking account of the effect of the liquid compressibility.

\section{REFERENCES}

1. Kurokawa, J., K. Kamijo, and T. Shimura. 1994. Axial thrust behavior in LOXpump of rocket engine. J. Propul. Power 10(2):244-50.

2. Abe, H., K. Matsumoto, J., Kurokawa, J. Matsui, and Y. Choi. 2006. Analysis and control of axial thrust in centrifugal pump by use of J-Groove. 23rd IAHR Symposium. Yokohama.

3. Shimura, T., S. Kawasaki, M. Uchiumi, and J. Matsui. 2011. Internal flow and axial thrust balancing of a rocket pump. ASME-JSME-KSME Joint Fluids Engineering Conference Proceedings. Hamamatsu, Japan. AJK2011-06027.

4. Della Gatta, S., S. Sslvadori, P. Adami, and L, Bertolazzi. 2006, CFD study for assessment of axial thrust balance in centrifugal multistage pumps. Conference on Modelling Fluid Flow (CMFF'06), The 13th Conference (International) on Fluid Flow Technologies Proceedings. Budapest, Hungary.

5. Hooser, K. V., J. Bailey, and A. Majumda. 1999. Numerical prediction of transient axial thrust and internal flows in a rocket engine turbopump. AIAA Paper No. 992189 .

6. Childs, D. W. 1991. Fluid-structure interaction forces at pump-impeller-shroud surfaces for axial vibration analysis. Trans. ASME, J. Vibr. Acoustics 113:108-15.

7. Hsu, Y., and C.E. Brennen. 2002. Fluid flow equations for rotordynamic flows in seals and leakage paths. Trans. ASME, J. Fluids Eng. 124(1):176-81.

8. Horiguchi, H., Y. Ueno, K. Takahashi, K. Miyagawa, and Y. Tsujimoto. 2009. Dynamic characteristics of the radial clearance flow between axially oscillating rotational disk and stationary disk. Int. J. Fluid Mach. Syst. 2(2):147-55.

9. http://www.Imsintl.com/. 2012.

10. Rhote-Vaney, R., V. Thomas, and A. Lekeux. 2002. Transient modelling of cryogenic rocket engines: A modular approach. 4th Conference (International) on Launcher Technology Space Launcher Liquid Propulsion. Liège, Belgium. 